\title{
A Fatal Association of COVID-19 and Acute Type A Aortic Dissection
}

\author{
Rana Irilouzadian ${ }^{1}$, Hossein Salehi Omran $^{1}$, and Toktam Alirezaei ${ }^{1}$ \\ ${ }^{1}$ Shahid Beheshti University of Medical Sciences
}

December 15, 2021

\begin{abstract}
We represent a case with simultaneous COVID-19 and acute type A aortic dissection. Type A aortic dissection, an aortic catastrophic event, seems to have higher mortality on coexistence with COVID-19.
\end{abstract}

\section{A Fatal Association of COVID-19 and Acute Type A Aortic Dissection}

Rana Irilouzadian ${ }^{1}$, Hossein Salehi Omran ${ }^{1}$, Toktam Alirezaei ${ }^{2 *}$

Faculty of medicine, Shahid Beheshti University of Medical Sciences, Tehran, Iran, emails: ranairilouzadian@gmail.comORCID: 0000-0001-8849-329X,hosseinsalehiomran@gmail.com

Cardiology Department of Shohadaye-Tajrish Hospital, Shahid Beheshti University of Medical Sciences, Tehran, Iran, emails: Alirezaei.toktam@sbmu.ac.irORCID: 0000-0002-4473-7093

*Author of correspondence: Toktam Alirezaei, email: Alirezaei.Toktam@sbmu.ac.ir

Address: Shohadaye-Tajrish Hospital, Tajrish sq., Tehran, Iran

Tel: +982125719

\section{Key Clinical Message}

We represent a case with simultaneous COVID-19 and acute type A aortic dissection. Type A aortic dissection, an aortic catastrophic event, seems to have higher mortality on coexistence with COVID-19.

Keywords: aortic dissection, COVID-19, coronavirus, cardiovascular disorders

As the patient was deceased, a written informed consent was obtained from the patient's next of kin to publish this report in accordance with the journal's patient consent policy and all of the authors declare that confidentiality of the patient was respected.

\section{Introduction}

Corona virus disease 2019 (COVID-19) is a respiratory disease caused by novel corona virus, severe acute respiratory syndrome coronavirus 2 (SARS-COV-2), which was first reported in Wuhan city of China on December 31, 2019 to world health organization ${ }^{1}$. Since then, more than 250 million people around the world have been infected and unfortunately more than five millions of them have lost their lives to date (November 2021) ${ }^{2}$. Covid-19 can present in a wide range of clinical manifestations, more commonly as respiratory symptoms such as shortness of breath and cough. Furthermore, it can manifest as cardiovascular symptoms such as palpitations and chest pain ${ }^{3}$ and cardiovascular complications such as aortic dissection 4. Aortic dissection is described as a tear in the intima layer of aortic wall which allows blood to flow and accumulate between aortic layers and can propagate along the length of aorta. It can manifest as various 
symptoms such as chest pain, weakness, fatigue and unequal extremity pulses and blood pressures ${ }^{5}$. Despite all advances in medicine in the field of diagnostics and treatments, aortic dissection has still a high rate of mortality which makes it extremely considerable ${ }^{6}$. In this report, we aim to present our patient with aortic dissection and recent COVID-19 and report her final destiny.

\section{Case history/examination}

A 46 years old woman was admitted to our emergency room on September 21st 2021, with acute onset chest pain which was retrosternal and radiating to the left arm and between two shoulders with a feeling of heaviness in the chest. The pain had a continuous nature from two hours before admission. In addition, she mentioned nausea, vomiting and cold sweat. The patient was nonsmoker and had a past medical history of stage 1 hypertension based on ACC/AHA 2021 hypertension guidelines for about three years and hyperlipidemia. She was under a single-drug treatment with a daily dosage of $25 \mathrm{mg}$ of losartan tablet for hypertension and was under control. She was also affected by COVID-19 three weeks ago and underwent outpatient treatment with remdesevir for five days during which the patient didn't experience fluctuations in blood pressure.

On physical examination, a systolic blood pressure difference of $40 \mathrm{mmHg}$ between arms $(140 / 80 \mathrm{mmHg}$ in right arm versus $100 / 80 \mathrm{mmHg}$ in left arm) was detected by sphygmomanometer in the assessment of vital signs. Her heart rate was 82 beats per minute. Respiratory rate was 16 per minute and arterial O2 saturation was $96 \%$. Pulmonary and heart auscultation were unremarkable. A discrepant pulse between left and right radial artery was felt which the left radial pulse was weaker. Routine neurological examination was normal, and the power in all limbs was $5 / 5$, with a Glasgow coma scale of $15 / 15$. Pupils were $3 \mathrm{~mm}$ bilaterally equal and reactive to light, the abdomen was soft and lax with no tenderness.

\section{Differential diagnosis, investigations and treatment}

Initial ECG showed normal sinus rhythm, normal axis with ST elevation in aVR and V1 leads and ST depression in I, aVL, V4-6 leads (Figure 1). According to patient's presentation with chest pain, unequal radial pulses, a difference in blood pressures of arms and her ECG, aortic dissection was the most likely diagnosis and aortic computed tomography (CT) angiography was requested for the patient.

In laboratory tests, D-dimer was $4820 \mathrm{ng} / \mathrm{ml}$ (reference up to 600), serum ferritin $417.4 \mathrm{ng} / \mathrm{ml}$ (reference 10291), lactate dehydrogenase $666 \mathrm{U} /$ lit (reference 230-480), troponin $0.2 \mathrm{ng} / \mathrm{ml}$ (normal population < 0.05), CPK 58 U/lit (24-170), CK-MB 20 U/lit (reference 1-24), C-reactive protein $16 \mathrm{mg} /$ lit (reference up to 5.9), ESR $26 \mathrm{~mm} / \mathrm{h}$ (reference up to 20 in below 50 years old patients). Other para-clinical tests were normal.

Following aortic CT angiography, aortic dissection type A according to Stanford classification and type 1 according to DeBakey classification was diagnosed for the patient.

The intimal flap started at aortic valve extending to ascending aorta, aortic arch, descending thoracic aorta, abdominal aorta to right common iliac artery and then terminating in right external iliac artery. Dissection flap extension to right carotid artery, right common carotid artery and left subclavian artery were noted (Figure 2). Right coronary artery was supplied by true lumen and left main artery was supplied by false lumen. The chest CT scan showed patchy ground glass opacity in anterior segment of superior and middle lobes of right lung consistent with COVID-19 infection.

Due to the diagnosis of aortic dissection, the patient was candidate for emergency surgery and due to inadequate facilities, she was prepared to be transferred to another hospital, but as soon as she entered the ambulance, she became bradycardic, leading to asystole. Cardiopulmonary resuscitation was performed and she was returned to the first hospital. The patient underwent intubation and atropine injection, after which the patient was successfully recovered after a few minutes and she regained consciousness. After an hour, she was transferred to another hospital for emergency surgery.

\section{Outcome and follow-up}

The patient was unfortunately expired before entering the operating room. 


\section{Discussion}

In this report, we described a 46 year old woman with past medical history of stage 1 hypertension which was under control, hyperlipidemia and recent COVID-19 whom represented with acute chest pain and cold sweat. The patient reported no traumatic events and no history of connective tissue disorders. Her clinical examination showed unequal radial pulses and a blood pressure difference between arms. During patient's hospitalization, her blood pressure was within the normal range. The laboratory tests showed elevated D-dimer, troponin, ESR and CRP. The diagnosis and management of aortic dissection in early stages is of importance since it has numeric manifestations and can mimic other life threatening events such as myocardial infarction and pulmonary embolism ${ }^{7}$. Silvestri et al., reviewed seventeen cases of aortic pathology in patients with clinically suspected or PCR confirmed COVID-19 and also reported hypertension as the most frequent comorbidity; they suggested a potential link between COVID-19 and aortic dissection ${ }^{8}$.

There are some potential mechanisms for arterial pathology in COVID-19 patients. SARS-CoV-2 has spike proteins on its surface that binds a receptor which is expressed in the endothelium called angiotensin converting enzyme 2 (ACE-2). This means that SARS-CoV-2 can injure vascular endothelium in the body 9 . SARS-CoV-2 downregulates ACE-2 which leads to over activation of classical renin-angiotensin system (RAS) and vasoconstriction ${ }^{10}$. ACE Inhibitor (ACEI) and angiotensin receptor blocking (ARB) drugs, which are used commonly for hypertension as in our patient, upregulate ACE-2 expression that can potentially increase the vascular entry of and injury by SARS-CoV-2. On the other hand, upregulation of ACE-2 can have vasodilatory and anti-inflammatory effects as a result of conversion of angiotensin II to angiotensin 1-7 $7^{11}$. However, in a study of 1128 hospitalized patients with COVID-19, those who took ACEI/ARB drugs had a lower all-cause mortality than those who didn't take ${ }^{12}$.

Another possible cause of arterial dissection in COVID-19 patients can be cytokine storm and inflammatory responses which leads to endothelial dysfunction ${ }^{13}$. Inflammation may cause rupture of atherosclerotic plaque which can lead to dissection ${ }^{14}$. Studies have shown that the number of patients with aortic dissection were increased during the influenza season ${ }^{15,16}$. Akgul et al., presented an aortic dissection in a COVID-19 patient which during the aortomy, they noticed significant aorta wall thickening as seen in inflammatory aortic pathologies ${ }^{17}$. Their finding is consistent with the potential association of inflammation caused by SARS-CoV-2 with aortic dissection.

As it has been suggested before, SARS-CoV-2 is a virus that causes multi-organ diseases and can manifests as life-threatening events ${ }^{10}$. Therefore, it is important to evaluate the association between COVID-19 and aortic dissection and the pathophysiology of it. Further studies are needed to establish this association.

\section{Conflict of interest}

The authors have no conflict of interests to declare.

\section{Acknowledgement}

The authors of the present study sincerely thank all of the medical staff in Shohadaye-Tajrish hospital, Tehran, Iran, who cooperated with us for completion of this study. All of the data in this report was extracted from the patient's hospital medical records.

\section{Ethical statement}

As the patient was deceased, a written informed consent was obtained from the patient's next of kin. All of the authors declare that confidentiality of the patient was respected.

\section{Funding}

There is no funding to the present study.

\section{Author's contribution}

Rana Irilouzadian: conceptualization, writing original draft, review and editing 
Hossein Salehi Omran: data collection, writing original draft

Toktam Alirezaei: data curation, supervision, review and editing

\section{References}

1. Ciotti M, Ciccozzi M, Terrinoni A, Jiang WC, Wang CB, Bernardini S. The COVID-19 pandemic. Crit Rev Clin Lab Sci . Sep 2020;57(6):365-388. doi:10.1080/10408363.2020.1783198

2. WHO COVID-19 Dashboard. Geneva: World Health Organization, 2020. Available online: https://covid19.who.int/ (last cited: [November 12,2021])

3. Baj J, Karakuła-Juchnowicz H, Teresiński G, et al. COVID-19: Specific and Non-Specific Clinical Manifestations and Symptoms: The Current State of Knowledge. $J$ Clin Med . Jun 5 2020;9(6)doi:10.3390/jcm9061753

4. Fukuhara S, Tang H, Kim KM, et al. Type A Aortic Dissection During COVID-19 Pandemic: Report From Tertiary Aortic Centers in the United States and China. Semin Thorac Cardiovasc Surg. Summer 2021;33(2):303-312. doi:10.1053/j.semtcvs.2020.10.034

5. Patel PD, Arora RR. Pathophysiology, diagnosis, and management of aortic dissection. Ther Adv Cardiovasc Dis . Dec 2008;2(6):439-68. doi:10.1177/1753944708090830

6. Karthikesalingam A, Holt PJ, Hinchliffe RJ, Thompson MM, Loftus IM. The diagnosis and management of aortic dissection. Vasc Endovascular Surg. Apr 2010;44(3):165-9. doi:10.1177/1538574410362118

7. Volvovitch D, Ram E, Cohen H, Kogan A, Sternik L, Raanani E. Acute pulmonary embolism following acute type A aortic dissection in a patient with COVID-19. Journal of Cardiac Surgery . 2021;36(4):15661568. doi:https://doi.org/10.1111/jocs.15389

8. Silvestri V, Recchia GE. Aortic Pathology During COVID - 19 Pandemics. Clinical Reports in Literature and Open Questions on the two Co-Occurring Conditions. Annals of Vascular Surgery . 2021;75:109-119. doi:10.1016/j.avsg.2021.02.037

9. Zubair AS, McAlpine LS, Gardin T, Farhadian S, Kuruvilla DE, Spudich S. Neuropathogenesis and Neurologic Manifestations of the Coronaviruses in the Age of Coronavirus Disease 2019: A Review. JAMA Neurology . 2020;77(8):1018-1027. doi:10.1001/jamaneurol.2020.2065

10. Divani AA, Andalib S, Di Napoli M, et al. Coronavirus Disease 2019 and Stroke: Clinical Manifestations and Pathophysiological Insights.J Stroke Cerebrovasc Dis . 2020;29(8):104941-104941. doi:10.1016/j.jstrokecerebrovasdis.2020.104941

11. Guo J, Huang Z, Lin L, Lv J. Coronavirus Disease 2019 (COVID-19) and Cardiovascular Disease: A Viewpoint on the Potential Influence of Angiotensin-Converting Enzyme Inhibitors/Angiotensin Receptor Blockers on Onset and Severity of Severe Acute Respiratory Syndrome Coronavirus 2 Infection. Journal of the American Heart Association . 2020;9(7):e016219-e16219. doi:10.1161/JAHA.120.016219

12. Zhang P, Zhu L, Cai J, et al. Association of Inpatient Use of Angiotensin-Converting Enzyme Inhibitors and Angiotensin II Receptor Blockers With Mortality Among Patients With Hypertension Hospitalized With COVID-19. Circ Res . 2020;126(12):1671-1681. doi:10.1161/CIRCRESAHA.120.317134

13. Markus HS, Brainin M. COVID-19 and stroke-A global World Stroke Organization perspective. International Journal of Stroke . 2020;15(4):361-364. doi:10.1177/1747493020923472

14. Oz K, Iyigun T, Karaman Z, et al. Prognostic Value of Neutrophil to Lymphocyte Ratio and Risk Factors for Mortality in Patients with Stanford Type A Aortic Dissection. Heart Surg Forum. Jun 30 2017;20(3):E119-e123. doi:10.1532/hsf.1736

15. Mehta RH, Manfredini R, Bossone E, et al. The winter peak in the occurrence of acute aortic dissection is independent of climate. Chronobiol Int . 2005;22(4):723-9. doi:10.1080/07420520500179605 
16. Ashur C, Norton E, Farhat L, et al. Higher admission rates and in-hospital mortality for acute type A aortic dissection during Influenza season: a single center experience. Sci Rep. Mar 13 2020;10(1):4723. doi:10.1038/s41598-020-61717-5

17. Akgul A, Turkyilmaz S, Turkyilmaz G, Toz H. Acute Aortic Dissection Surgery in a Patient With COVID-19. Ann Thorac Surg . Jan 2021;111(1):e1-e3. doi:10.1016/j.athoracsur.2020.06.005

\section{Images, graphs and videos}

Figure 1. Initial ECG of patient: normal sinus rhythm, normal axis, ST elevation in aVR and V1 leads and ST depression in I, aVL, V4-6 leads

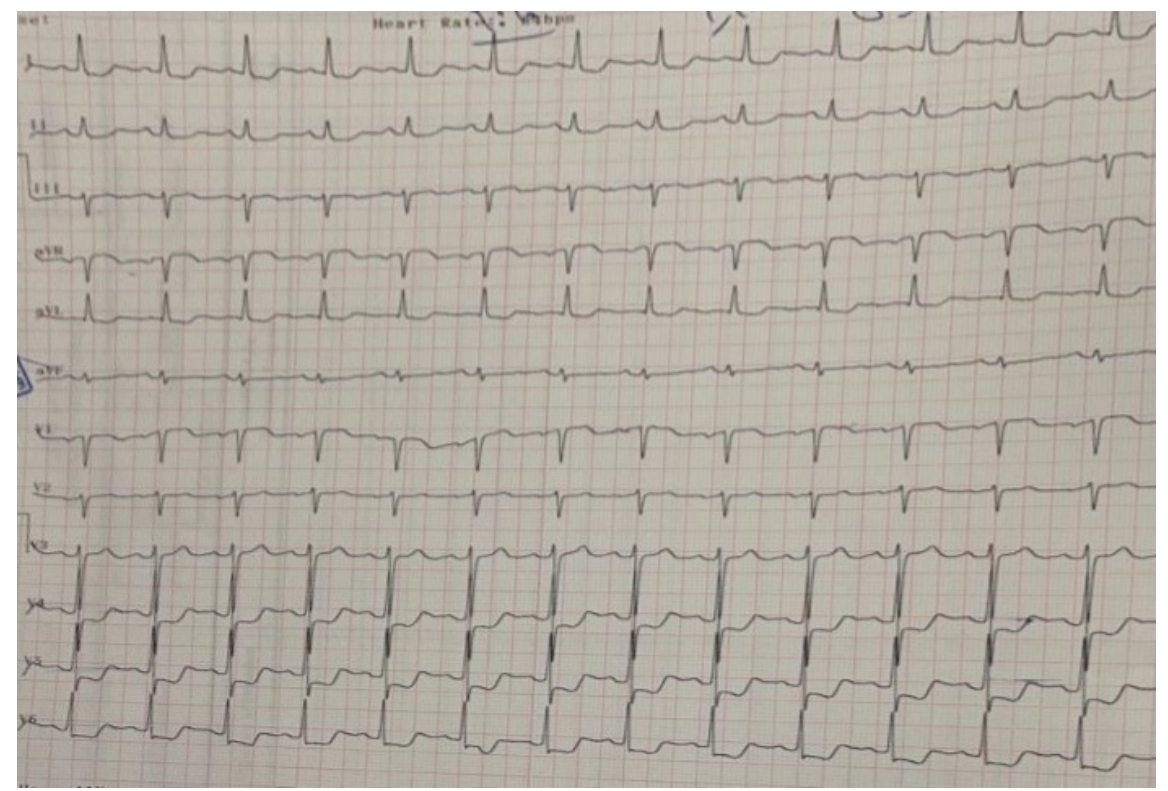

Figure 2. Aortic CT angiography: type A according to Stanford classification and type 1 according to DeBakey classification

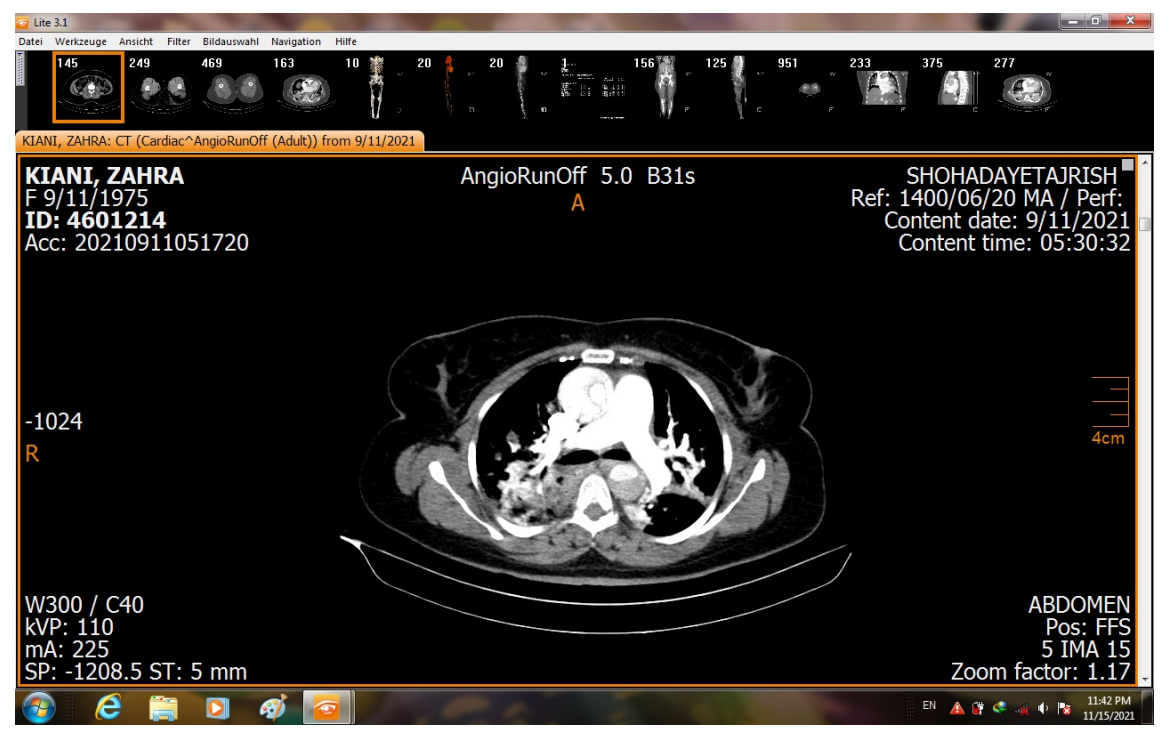




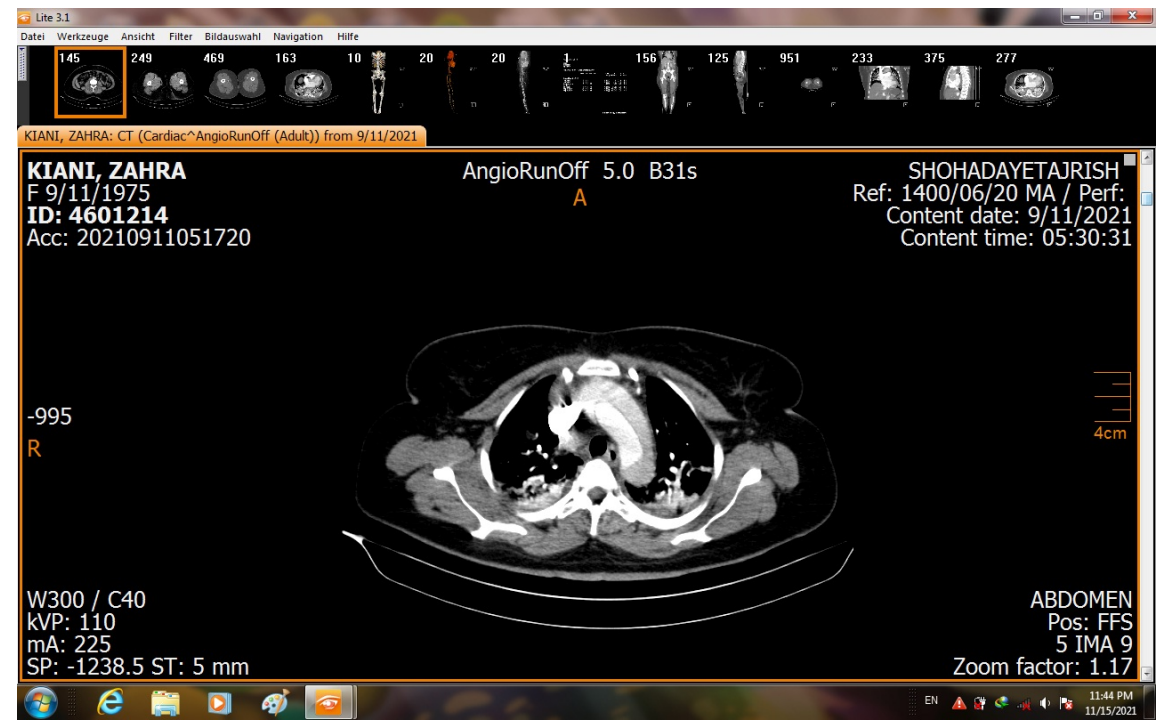

